\title{
Strongly Semistable Bundles on a Curve over a finite field S. Subramanian
}

\begin{abstract}
We show that a principal $\mathrm{G}$ bundle on a smooth projective curve over a finite field is strongly semistable if and only if it is defined by a representation of the fundamental group scheme of the curve into G.
\end{abstract}

MSC 2000 classification:14-XX

Keywords:semistable bundles,fundamental group scheme.

\section{Introduction}

Let $G$ be a semisimple algebraic group and $X$ a smooth projective curve, both defined over a finite field $k$ of characteristic $p$. Let $E \rightarrow X$ be a principal $G$-bundle on $X . E$ is said to be stable (resp. semistable) if for every reduction of structure group $E_{P} \subset E$ to a parabolic subgroup $P$ of $G$, we have

$$
\operatorname{deg} E_{P}(\mathfrak{p})<(\text { resp. } \leq) \operatorname{deg} E_{G}(\mathfrak{g})
$$

where $\mathfrak{g}$ and $\mathfrak{p}$ denote the lie algebras of $G$ and $P$ respectively,and $E_{P}(\mathfrak{p})$ (respectively $\left.E_{G}(\mathfrak{g})\right)$ denotes the Lie algebra bundle associated to $E_{P}$ (respectively $E_{G}$ ) by the adjoint action of $P($ respectively $G)$ on $\mathfrak{p}$ (respectively $\mathfrak{g}$ ). When the base 
field is the field of complex numbers, the theorem of Narasimhan-SeshadriRamanathan says that a stable $G$-bundle is defined by a unitary representation of the topological fundamental group of $X$. In this article, we show that in the case when $k$ is a finite field, a strongly semistable principal $G$-bundle on $X$ (recall that a bundle is said to be strongly semistable if all of its Frobenius pull backs are semistable) is defined by a representation of the fundamental group scheme $\pi(X) \rightarrow G$. Here, by fundamental group scheme, we mean the proalgebraic group scheme introduced by M. Nori in [3], [4]. The author wishes to thank the referee for his helpful comments on an earlier version of this paper.

\section{Preliminaries}

Let $k$ be a finite field of characteristic $p$ and let $X$ be a smooth projective curve defined over $k$. We assume that $X$ has a rational point (say $x$ ) defined over $k$. A vector bundle $V \rightarrow X$ (over $k$ ) of degree zero is said to be essentially finite if there exists a finite group scheme $H$ over $k$ and a principal $H$-bundle $\pi: E_{0} \rightarrow X$ on $X$, such that $\pi^{*} V$ is trivial on $E_{0}$ (see Proposition(3.10),page 83,in [5]). Let $\mathcal{C}$ denote the category of all essentially finite vector bundles defined on $X$ over $k$. We define a fibre functor $T$ on $\mathcal{C}$ by defining $T(V)=V_{x}$, the fibre of $V$ at $x$, for every $V \in \mathcal{C}$. With this fibre functor, $\mathcal{C}$ is a Tannaka category over $k$ (see section 2.3 in [5]). The associated affine group scheme $\pi(X, x)$ is called the fundamental group scheme of $X$ over $k$. Further, there is a principal $\pi(X, x)$ bundle $\mathcal{X} \rightarrow X$ over $k$ (see section 2.3,page 84,in

[5]). Given a semisimple algebraic group $G$ over $k$, and an algebraic group 
homomorphism $\pi(X, x) \rightarrow G$ (such a homomorphism factors through a finite group scheme quotient of $\pi(X, x))$, there is associated a principal $G$-bundle $E \rightarrow X$, associated to $\mathcal{X} \rightarrow X$ by the homomorphism $\pi(X, x) \rightarrow G$.

We now consider a principal $G$ bundle $E \rightarrow X$, which we assume to be strongly semistable. Since $G$ is assumed to be semisimple, we can find a $G$-module $V$ such that $G \subset S L(V)$. The associated vector bundle $E(V)$ is strongly semistable by the theorem of Ramanan-Ramanathan (see Theorem 3.23 in [6] ). We form the category $\mathcal{M}$ whose objects are subquotients of degree zero of finite direct sums

$$
\bigoplus_{i} E(V)^{\oplus n_{i}} \oplus E(V)^{* \oplus m_{i}}
$$

where $n_{i}, m_{i}$ are nonnegative integers. The homomorphisms in $\mathcal{M}$ are all homomorphisms of the respective vector bundles on $X$. Given a vector bundle $W$ in $\mathcal{M}$, we can associate to it its fibre $W_{x}$ at $x$, and with this as the fibre functor, $\mathcal{M}$ is a Tannaka category over $k$ (see Section 4 in [1]). There is an associated affine group scheme $M$, called the monodromy group scheme of $E$.Further,if $V \operatorname{ect}(X)$ denotes the category of vector bundles on $X$, then there is a naturally defined functor $\phi: \mathcal{M} \rightarrow V e c t(X)$ which associates to an object of $\mathcal{M}$ the vector bundle on $X$ that it represents. The functor $\phi$ satisfies the axioms of Lemma(2.1),page 77, in [5].Hence,by Proposition(2.5),page 78,in [5],we obtain a principal $M$-bundle $E_{M} \rightarrow X$ on $X$, defined over $k$ (see [1],section 4). Further, since $k$ is a finite field, $H^{1}(k, G)$ is trivial (see Theorem 1',section 2.3, Chapter 3,page 132 in [7]), and hence we can assume that there is a $k$ rational point in the fibre $E_{x}$ of $E$ at $x$. With this choice of point in $E_{x}$, we obtain an embedding $M \subset G$, and a reduction of structure group $E_{M} \subset E$ (see section 4 in [1] for details). The monodromy group 
scheme $M$ and the bundle $E_{M}$ do not depend on the choice of representation $G \subset S L(V)$, and the bundle $E_{M}$ is characterised as the minimal degree zero reduction of structure group of $E$ (see Proposition 4.9, Corollary 4.10 and Proposition 4.11 in [1]).

\section{Main Theorem}

Let $k$ be a finite field and let $X$ be a smooth projective curve over $k$. We assume that $X$ has a rational point $x$ over $k$. Let $G$ be a semisimple algebraic group over $k$. Let $F$ denote the Frobenius morphism on $X$.

Definition (3.1) A principal $G$ bundle $E \rightarrow X$ on $X$ over $k$ is said to be semistable if, for every reduction of structure group $E_{P} \subset E$ to a parabolic subgroup $P$ of $G$, we have

$$
\text { degree } E_{P}(\mathfrak{p}) \leq 0 \text {. }
$$

The principal bundle is said to be strongly semistable if $F^{n^{*}} E$ is semistable for all $n \geq 1$. We can now prove

Theorem (3.2) Let $E \rightarrow X$ be a strongly semistable $G$-bundle on $X$ over $k$. Then $E \rightarrow X$ is defined by a homomorphism $\pi(X, x) \rightarrow G$. This homomorphism $\pi(X, x) \rightarrow G$ is unique upto inner conjugation by an element of $G(k)$. Further, if $G^{\prime}$ is another semisimple algebraic group over $k$ and $G \rightarrow G^{\prime}$ a homomorphism of algebraic groups, then the strongly semistable $G^{\prime}$ bundle $E_{G^{\prime}} \rightarrow X$ associated to $E \rightarrow X$ is defined by the composite homomorphism $\pi(X, x) \rightarrow G \rightarrow G^{\prime}$. 
Proof. By hypothesis, the bundles $F^{n *} E, n \geq 1$ are all semistable. Since $k$ is a finite field, by boundedness, the set $\left\{F^{n *} E, n \geq 1\right\}$ is a finite set. Hence $F^{n *} E \cong F^{(n+\ell) *} E$ for some positive integers $n$ and $\ell$. We now consider the monodromy group scheme $M_{n}$, and the monodromy bundle $E_{M_{n}}$, of the bundle $F^{n *} E$ for $n \geq 0$ (with the convention that $F^{0 *} E=E$ ). Let $F_{n}$ denote the Frobenius morphism of the group scheme $M_{n}$. The isomorphism $F^{n *} E \cong F^{(n+\ell) *} E$ implies that $F_{n}^{\ell}\left(M_{n}\right) \cong M_{n+\ell} \cong M_{n}$, and further the monodromy bundles $E_{M_{n}}$ and $E_{M_{n+\ell}}$ are isomorphic. We now observe that the isomorphism

$$
F_{n}^{\ell}\left(M_{n}\right) \cong M_{n+\ell} \cong M_{n}
$$

implies that the group scheme $M_{n}$ is finite and reduced.Then by Lemma (4.12) in [1], it follows that $M_{0, r e d}$, the reduced part of the group scheme $M_{0}$ (where $M_{0}$ denotes the monodromy group scheme of the bundle $E$ ), is isomorphic to $M_{n}$. Also, if $F_{0}$ denotes the Frobenius morphism of $M_{0}$, we obtain $F_{0}^{n}\left(M_{0}\right) \cong M_{n}$. Hence $M_{0}$ is a finite group scheme over $k$. The monodromy bundle $E_{M_{0}}$ is a principal $M_{0}$ bundle on $X$, and the reduction of structure group $E_{M_{0}} \subset E$ implies that the pull back of the bundle $E$ to $E_{M_{0}}$ is trivial. The principal $M_{0}$ bundle $E_{M_{0}} \rightarrow X$ defines a quotient $\pi(X, x) \rightarrow M_{0}$ (see [5]), and the composite homomorphism

$$
\pi(X, x) \rightarrow M_{0} \rightarrow G
$$

is the homomorphism we want defining the principal $G$-bundle $E$.

For the second part, let $G \rightarrow G^{\prime}$ be a homomorphism of semisimple groups over $k$. Since $G$ is semisimple, there are three cases to consider:

Case (i) $G \subset G^{\prime}$ is a closed immersion: in this case, a faithful representation of 
$G^{\prime}$ is also a faithful representation of $G$, and the result follows from the fact that the monodromy group scheme and the monodromy bundle of $E$ are independent of the choice of representation.

Case (ii) $G \rightarrow G^{\prime}$ is a central isogeny: we consider the case $G^{\prime}=G / Z$, where $Z$ is the centre of $G$. Let $M$ be the monodromy group scheme of $E$ and $E_{M}$ the monodromy bundle. Let $M^{\prime}$ be the image of $M$ in $G^{\prime}$ and $E_{M^{\prime}}$ the bundle associated to $E_{M}$ by the projection $M \rightarrow M^{\prime}$. Then $E^{\prime}$ has a reduction of structure group $E_{M^{\prime}} \subset E^{\prime}$ to $M^{\prime}$, of degree zero(see Definition 4.7 in [1]). If $H$ is a group scheme over $k$ and $E_{H} \subset E^{\prime}$ a reduction of structure group of $E^{\prime}$ of degree zero to $H$, with $H \subset M^{\prime}$, then taking $\widetilde{H}$ to be the inverse image of $H$ in $G$, we obtain a principal $\widetilde{H}$ bundle $E_{\widetilde{H}}$ and a reduction of structure group $E_{\widetilde{H}} \subset E$ of degree zero with $\widetilde{H} \subset M$. This contradicts the minimality of $M$ and $E_{M}$ (see Corollary (4.10) in [1]). It follows that $M^{\prime}$ is the minimal subgroup scheme of $G^{\prime}$ with a degree zero reduction of structure group to $M^{\prime}$. Hence, again by Corollary (4.10) in [1], it follows that $M^{\prime}$ is the monodromy group scheme of $E^{\prime}$ and $E_{M^{\prime}}$ is the monodromy bundle of $E^{\prime}$.

Case(iii) $G \rightarrow G^{\prime}$ is purely inseparable:to treat this case,it is enough to consider the Frobenius morphism $F: G \rightarrow G$,as the general case is what is usually called a Frobenius sandwich.Given the principal G bundle $E \rightarrow X$, the bundle induced by the Frobenius $F: G \rightarrow G$ is the pullback $F^{*} E$ (where the pullback is taken under the Frobenius morhism of $X$ ).Let $M$ be the monodromy groupscheme of $E$ and $E_{M} \subset E$ the 
monodromy reduction. We know that $M$ is a finite groupscheme.Let $F_{M}$ denote the Frobenius morphism of $M$ and let $M^{\prime}$ be the image of $M$ under $F_{M}$. The $M$ bundle $E_{M}$ induces a $M^{\prime}$ bundle $E_{M^{\prime}}$ and a reduction of structure group $E_{M^{\prime}} \subset F^{*} E$.If there exists a proper subgroupsheme $N \subset M^{\prime}$ and a reduction of structure group $E_{N} \subset E_{M^{\prime}}$, then the inverse image of $E_{N}$ under the projection $E_{M} \rightarrow E_{M^{\prime}}$ would define a reduction of structure group of $E_{M}$,contradicting the minimality of $E_{M}$. Therefore $E_{M^{\prime}}$ is a minimal reduction of structure group of $F^{*} E$, and hence is the monodrmy reduction.

It now follows that the monodromy of the bundle $E^{\prime}$ is given by the composite homomorphism $\pi(X, x) \rightarrow G \rightarrow G^{\prime}$ Q.E.D.

\section{When $X$ does not have a rational point}

Let $X$ be a smooth projective curve over a finite field $k$ as before and let $E \rightarrow X$ be a principal $\mathrm{G}$ bundle on $X$ which is strongly semistable.Let $l$ be a finite Galois extension of $k$ over which $X$ acquires a rational point.Let $X_{l}$ denote the base change of $X$ to $\operatorname{Spec}(l)$ and $E_{l}$ the base change of $E$ to $\operatorname{Spec}(l)$. Then by section 3 above, $E_{l}$ is defined by a homomorphism $\pi\left(X_{l}\right) \rightarrow$ $G_{l}$,where $\pi\left(X_{l}\right)$ denotes the fundamental group sheme of $X_{l}$ and $G_{l}$ denotes the base change of $G$ to $\operatorname{Spec}(l)$. We have the following exact sequence of group schemes over $\operatorname{Spec}(k)$,

$$
1 \rightarrow \pi\left(X_{l}, x\right) \rightarrow \pi(X, x) \rightarrow \operatorname{Gal}(l / k) \rightarrow 1
$$

where $x$ is a closed point of $X$ with residue field $l$,so it is a rational point over $l, \pi$ is the fundamental group scheme, and $\operatorname{Gal}(l / k)$ is the Galois group 
of $l$ over $k$.Here $\pi\left(X_{l}, x\right)$, which is a group scheme over $\operatorname{Spec}(l)$,is regarded as a group scheme over $\operatorname{Spec}(k)$ by the projection $\operatorname{Spec}(l) \rightarrow \operatorname{Spec}(k)$. The homomorphism $\pi\left(X_{l}, x\right) \rightarrow G_{l}$ factors through a finite group scheme quotient, say $H$,of $\pi\left(X_{l}, x\right)$.Since $E_{l}$ is actually defined over $k$,it follows that the monodromy group scheme $M$ of $E_{l}$, and the monodromy reduction $E_{M} \subset E_{l}$, remain invariant under the action of $G a l(l / k)$ on $E_{l}$.Hence the subgroupscheme $H \subset G_{l}$ remains invariant under the action of $G a l(l / k)$.Every groupsheme quotient of $\pi\left(X_{l}, x\right)$ invariant under the action of $\operatorname{Gal}(l / k)$ on $\pi\left(X_{l}, x\right)$ descends to a quotient of $\pi(X, x)$.Hence $H$ is a quotient of $\pi(X, x)$ and we obtain the homomorphism $\pi(X, x) \rightarrow G$ defining $E$ on $X$ over $k$.

\section{References}

1. Indranil Biswas, A.J. Parameswaran and S. Subramanian: Monodromy group for a strongly semistable principal bundle over a curve, Duke Math. J. 132, No.1, 1-48 (2006).

2. H.Esnault,P.H.Hai,and X.Sun,On Nori's fundamental group scheme, arXiv:math.AG/0605645

3. H. Lange and U. Stuhler: Vectorbundel auf Kurven und Darstellungen der algebraischen Fundamentalgruppe, Math. Z. 156, No.1, 73-83 (1977).

4. M.V. Nori: On the representations of the fundamental group scheme, Comp. Math., 33, 29-41 (1976). 
5. M.V. Nori: The fundamental group scheme, Proc. Ind. Acad. Sci. (Math. Sci.), 91, 73-122 (1982).

6. S.Ramanan and A.Ramanathan:Some remarks on the instability flag, Tohoku Math.J.(2),36,269-291(1984).

7. J.P. Serre: Galois cohomology,English translation by P.Ion,Springer,1997

School of Mathematics

Tata Institute of Fundamental Research

Homi Bhabha Road, Colaba

Mumbai 400005

India

E-mail:subramnn@math.tifr.res.in 